\title{
Pressure Index in Cricket
}

\author{
Dr. Parag Shah, Mitesh Shah \\ 1. H L College of Commerce, Ahmedabad \\ 2. SV Arts College, Ahmedabad
}

\begin{abstract}
Usually, evaluation of individual player performances in one-day cricket has been based on measures such as batting and bowling averages, and strike and economy rates. It is accepted, within the game of cricket, that such measures have severe limitations in assessing the true performances and abilities of players. Whenever a player's performance statistics are quoted, there is nearly always some verbal prerequisite that is necessary in order to place the statistics into context. Such comments include the stage of an innings a player usually bats or bowls, as this significantly affects a player's opportunity for scoring runs or taking wickets. Further, traditional measures do not allow the comparison of the skills of batting and bowling as they are based on incompatible scales.

We have developed a new measure - Pressure Index. It measures the pressure under which the team is playing or a batsman is batting. Variables such runs scored, runs left, wickets, balls faced and balls left are considered for developing this new measure. Runs scored under more pressure are more valuable than runs scored under very less pressure. With this Pressure index actual performance of the batsman can be measured. Similar measure can be developed for bowlers.
\end{abstract}

Keywords: Pressure index, Current run rate, required run rate, Target.

\section{Introduction:}

Usually, evaluation of individual player performances in one-day cricket has been based on measures such as batting and bowling averages, and strike and economy rates. Various researchers like Machale(1990) and Lemmer (2006),(2008)have discussed various measures for measuring batting and bowling performances of a batsman and a bowler. It is accepted, within the game of cricket, that such measures have severe limitations in assessing the true performances and abilities of players.

Barr G.D.I. and Kantor B.S.(2004) have discussed a criterion for comparing and selecting batsman in limited over cricket and Kimber A.C. and Hansford A.R. (1993) has given a Statistical Analysis of Batting in Cricket. But it is recognized, within the game of cricket, that such measures have severe limitations in assessing the true performances and abilities of players.

Cricket has recently experienced a highly-successful intervention from the field of Operational Research in the form of the 'Duckworth-Lewis' method of adjusting target scores in one day matches Duckworth \& Lewis (1998) which was subsequently adopted by the International Cricket Council Duckworth \& Lewis (2004). Player performance remains of significant interest to cricket team selectors and coaches who are trying to build winning teams from a fixed pool of players or within a limited budget, and this imperative to win will probably lead to developments in the use of cricket statistics - at least among cricket professionals. Measuring cricketers only through their averages is liable to give skewed and unreliable results.

Lewis (2005) has tried to develop fairer measures of player performance in one-day cricket. But still whenever a player's performance statistics are quoted, there is nearly always some verbal prerequisite that is necessary in order to place the statistics into context. Such comments include the stage of an innings a player usually bats or bowls, as this significantly affects a player's opportunity for scoring runs or taking wickets. Further, traditional measures do not allow the comparison of the skills of batting and bowling as they are based on incompatible scales. That is these traditional measures do not include the stage of an innings a player usually bats or bowls, as this significantly affects a player's opportunity for scoring runs or taking wickets.

A typical T20 match has ups and downs: sometimes one thinks Team A is winning and at other times one thinks that Team B is more likely to win. This feeling about which side is winning is based on our general appreciation of an ODI game. If Team A is chasing a huge target and loses quick wickets you feel Team A is losing. If Team $\mathrm{A}$ is chasing a modest target and two opening batsmen are on the rampage, you feel Team $\mathrm{A}$ is winning. If Team A loses their openers while chasing 350, their pressure index might be high. If two openers blaze to 90 for no loss in 14 overs while chasing 210 in 50 overs, the pressure could be very low.

Can we quantify this feeling?

Can we come up with a number that measures the chance that the team chasing will win the match? 
We have developed a new measure - Pressure Index. It measures the pressure under which the team is playing or a batsman is batting. Variables such runs scored, runs left, wickets, balls faced and balls left are considered for developing this new measure. Runs scored under more pressure are more valuable than runs scored under very less pressure. With this Pressure index actual performance of the batsman can be measured. Similar measure can be developed for bowlers.

\section{Methodology}

In this paper we have defined the pressure index 'experienced' by a team chasing a target in anT20 match. If the match was evenly placed, the chasing team's pressure index would be exactly 100 . This index number fluctuate around 100, ranging from 0 to some high value above 100 . The logic was to look at the ratio of the required run rate and the current rate. Fall of wicket and the stage at which wicket falls should be considered in the index. That was the approximate idea: keep tracking the pressure index ball after ball! It was a great way to know at a glance which team was ahead, and by how much. The ball-by-ball pressure index could also be plotted graphically to produce a 'pressure graph'. This pressure graph provides a brilliant instantaneous assessment of how the ODI match went.

Using the historical data of last 150 T20 matches from website Cricinfo, we obtained the wicket weightage for our Pressure Index. From ball by ball data of a particular match we calculate current required run rate, initial required rate, balls faced, balls left, target, runs scored and from it Pressure Index is calculated ball by ball.

The formula for calculating Pressure Index (PI) is given below:

\section{$\mathbf{P I}=$ Req. rate/Initial $\mathbf{R R} * \mathbf{1 0 0}+$ \\ ((Wk. Wt./180)*Target)*((Total balls - balls faced $) /$ Total balls $) *$ \\ ((target - Runs scored)/Target)}

\section{Results}

To explain how pressure index and its graph is calculated ball by ball, we have considered 2007 T20 world cup final match between India and Pakistan. India batting first, scored 157 runs in 20 overs with batting average of 7.85 per over. Pakistan needed 158 runs to win in 20 overs. In other words Pakistan needed 1.32 runs per ball.

Table1 below shows ball by ball Pressure Index calculation for 18 balls and similar for rest of the balls can be calculated. We reproduce below the ball by ball pressure graph of Pakistan in this match.

Table 1: Ball by Ball Pressure Index for Pakistan

\begin{tabular}{|c|c|c|c|c|c|c|c|c|}
\hline 1 & 1 & 0 & 1 & 1.00 & 1.32 & 100.20 & 0.00 & 100.20 \\
\hline 2 & 0 & 0 & 1 & 0.50 & 1.33 & 101.05 & 0.00 & 101.05 \\
\hline 3 & 1 & 0 & 2 & 0.67 & 1.33 & 101.27 & 0.00 & 101.27 \\
\hline 6 & 2 & 0 & 4 & 0.67 & 1.35 & 102.60 & 27.07 & 129.67 \\
\hline 7 & 4 & 0 & 8 & 1.14 & 1.33 & 100.82 & 23.07 & 123.89 \\
\hline 8 & 6 & 0 & 14 & 1.75 & 1.29 & 97.65 & 17.07 & 114.72 \\
\hline 9 & 6 & 0 & 20 & 2.22 & 1.24 & 94.42 & 11.07 & 105.49 \\
\hline 10 & 0 & 0 & 20 & 2.00 & 1.25 & 95.28 & 11.07 & 106.35 \\
\hline 15 & 0 & 1 & 26 & 1.73 & 1.26 & 95.48 & 27.53 & 123.01 \\
\hline 16 & 0 & 0 & 26 & 1.63 & 1.27 & 96.40 & 27.53 & 123.93 \\
\hline 17 & 1 & 0 & 27 & 1.59 & 1.27 & 96.60 & 26.53 & 123.13 \\
\hline 18 & 4 & 0 & 31 & 1.72 & 1.25 & 94.56 & 22.53 & 117.09 \\
\hline . &. & . &. & . &. & . &. & . \\
\hline . &. & . & . & . &. & . &. & . \\
\hline$\cdot$ &. & . & . & . & 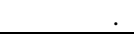 & 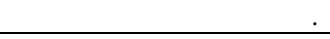 & . & . \\
\hline
\end{tabular}

*Additional Term here represents the calculation of remaining part of the formula. 


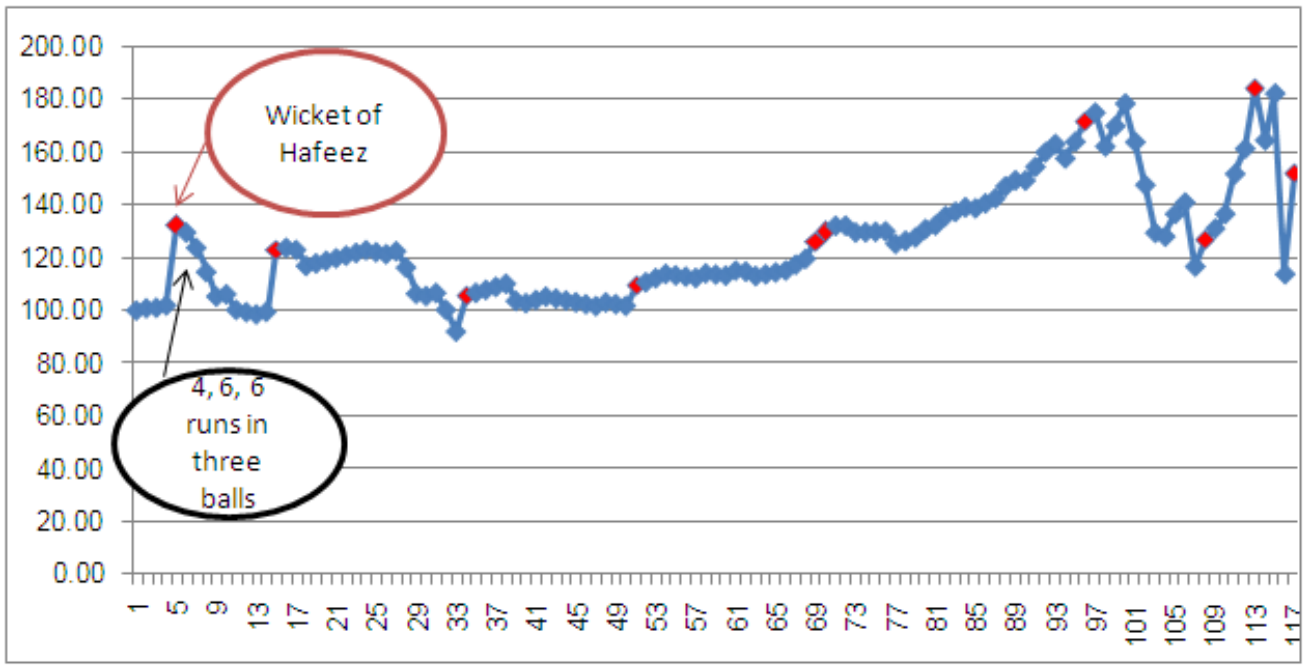

Pakistan starts with the required rate of 1.32 runs per ball. On the first ball they score 1 run which is less than required 1.32 runs so pressure index after first ball is 100.2. On the fifth ball Pakistan lost wicket of Hafeez, so the pressure index jumps to 132.10. Now on seventh, eighth and ninth ball, Pakistan scores 4, 6 and 6 runs, so the pressure index after ninth ball comes down to 105.49.

Pressure Index graph gives a perfect picture of fluctuating pressure. It shows how pressure index jumps up with a wicket and comes down with few big hits.

\section{Discussion}

Pressure index provides a clear situation of pressure in which a batsman is playing. This pressure index considers balls faced, balls left, runs scored, runs left, wicket, wickets left. Once the run made by the batsman is linked with the pressure index, it can be applied for various purposes. We can get an idea which batsman exactly performs under a particular pressure; this can help the team to set the batting order. Moreover the batsman's performance can be evaluated with one more cricketing measure - Pressure runs along with the average and strike rate. Runs scored by batsman who have performed well under pressure like Bevan and Dhoni value much higher than runs with relatively no pressure. This will identify a lot of players whose contributions often get overlooked because of usual cricket measures.

In this paper we have considered the pressure index only for team batting second. But pressure index for team batting first can be obtained by considering the average runs scored by a team batting first on that ground as target. Similar work can be done for the bowlers.

\section{References}

[1]. Barr, G.D.I. and Kantor, B.S. (2004).A criterion for comparing and selecting batsman in limited over cricket. Journal of Operation research Society, 55, 1266-1274.

[2]. Cricinfo: The home of cricket. http://www.cricinfo.com.

[3]. Duckworth, F. C. and Lewis, A. J. (1998). A fair method for resetting the target in interrupted one-day cricket matches. Journal of the Operational Research Society, 49(3), 220-227.

[4]. Duckworth, F.C. and Lewis, A.J. (2004). A successful operational research intervention in one-day cricket. Journal of the Operational Research Society, 55: 749-759.

[5]. Kimber, A.C. and Hansford, A.R. (1993 ).A Statistical Analysis of Batting in Cricket. J.R. Statist. Soc. A, 156, 3, 443-455

[6]. Lewis, A. J. (2005). Towards fairer measures of player performance in one-day cricket. Journal of the Operational Research Society, 56(7), 804-815.

[7]. Lemmer, H. H. (2006). A measure of the current bowling performance in cricket. South African Journal for Research in Sport, Physical Education and Recreation, 28(2), 91-103.

[8]. Lemmer, H. H. (2008). Measures of batting performance in a short series of cricket matches. South African Statistical Journal, 42(1), 65-87

[9]. Machale, D. (1990). What does "mean" mean? The Mathematical Gazette, 74(469), 239-243. 22 